in ihren Fällen den Bazillus nicht nachweisen; die Inokulation des Eiters bei Meerschweinchen hatte jedoch positiven Erfolg).

Da die von A. erhaltene Kultur die Anwesenheit von Staphylokokken in den Pusteln demonstrierte, konnte man sich fragen, welche pathogene Wirkung diesen und welche dem Tuberkelbazillus bei der Bildung der Pusteln zuzuschreiben war. Die experimentelle Impfung brachte keine volle Klarheit. Ein geimpftes Meerschweinchen starb schnell und zeigte pyämische Erscheinungen, die auf die in den kleinen Abszessen auffindbaren Staphylokokken bezogen werden konnten. Bei einem Kaninchen sah man schwere Alterationen der Leber, in denen nebeneinander sehr zahlreiche Kokken und Bazillen vorkamen. Bei zwei Meerschweinchen; die länger lebten, hatte die Tuberkulose das gewöhńliche Aüssehen der experimentellen Tuberkulose.

A. glaubt annehmen zu dürfen, daß der Ko chsche Bazillus das pathogenetische Agens der Pustel war, daß erst sekundär die Staphylokokken, die gewöhnlich bei den feuchten Krankheitsformen zu finden sind, hinzugetreten seien.

Man würde, meint er, auch 'schlecht verstehen, daß reïhliche Lokalisation und Vegetation von Tuberkelbazillen innerhalb einer durch vulgäre Eiterkokken hervorgerufenen Pustel ein sekundäres Faktum sei.

Die beschriebene Form von Folliculitis suppurativa vegetans, die für sich betrachtet, d. h. ohne andere konkomitierende schon bekannte tuberkulöse Läsionen, schwierig zu diagnostizieren ist, weil sie große Ähnlichkeit hat mit einer Folliculitis staphylococcica und in ihrer aggregierten Form mit einer Folliculitis trichophytica, ist auch der von Truffi unter dem Namen Folliculitis staphylogenes vegetans beschriebenen Affektion sehr ähnlich und ruft schließlich die Perifolliculitis agminata suppurativa (Ṕreis), ins Gedächtnis, deren pathologisches Agens ein azidophiler, dem Tuberkelbazillus ähnlicher Bazillus ist. J. Ullmann (Rom).

\title{
Polnische Zeitschrift für Haut- und venerische Krankheiten 1910. Band V. Nr. 9.
}

Krzysztalowicz, F. Die Plasmazellen (Unna). Nach Angabe der anfänglichen Definition Unnas der Plasmazellen, bespricht K. ihre Genese und Umänderungen in verschiedenen pathologischen Prozessen. Es unterliegt jetzt keinem Zweifel, daß die Plasmazelle ein rein pathologisches Gebilde und für gewöhnlich das Zeichen einer starken progressiven Ernährungsstörung ist. In verschiedenen pathologischen Prozessen begegnet man Bilder, welche die Entstehungsart der Plasmazellen aus den Bindegewebszellen, Endothelien und Perithelien der Blutgefäße illustrieren. Die Vermehrung vollzieht sich durch direkte Teilung, wodurch die sog. Plasmatochterzellen entstehen. Die Plasmazellen können bei 
diversen Krankheitsprozessen in der weiteren Entwicklung verschiedene Form einnehmen. Bei einigen Prozessen, wie Granulationen, lassen sich die Plasmazellen, infolge Ödems, weniger scharf von den Fibroblasten abtrennen, bei anderen, wie Lupus, Ulcus molle, Rhinophym, befinden sich anders aussehende Zellen, die Unna atrophische Plasmazellen benannt hat. Bei Mykosis fungoides beobachtet man die durch Granolyse und Chromatolyse veränderte Zellen, die auch künstlich mittelst der Behandlung des toten Materials durch Kochsalzlösungen oder durch die Einwirkung des Serums nachgeahmt werden können. Endlich erleiden die Plasmazellen bei einigen chronischen Dermatosen, wie beim Rhinosklerom, die hyaline Degeneration, wodurch sie verschiedene Formen einnehmen. Zuletzt erwähnt auch der Verf. die Meinungen verschiedener Autoren und Unnas selbst über die Herkunft der Plasmazellen. Diese versehiedenen Anschaungen und Hypothesen können aber die ursprüngliche Meinung Unnas, daß die Plasmazellen durch Proliferation der fixen Bindegewebszellen, der Endo- und Perithelien entstehen, bisher nicht stürzen, da wenigstens bis jetzt von niemandem mit absoluter Sicherheit bewiesen worden ist, daß Plasmazellen anderer Herkunft sind. Eine kolorierte Tafel illustriert alle beschriebenen Formen und Degenerationen der Plasmazellen. F, Krzysztalowicz (Krakau). 